\title{
SHION: Towards An Interactive Digital Twin Supporting Shopfloor Operations on Real Time
}

\section{F. J. Lacueva-Pérez}

Instituto Tecnológico de Aragón

S. Hermawati

University of Nottingham

\section{P. Amoraga}

Thermolympic, S.L.

R. Salillas

Instituto Tecnológico de Aragón

R. del Hoyo

Instituto Tecnológico de Aragón

G. Lawson

University of Nottingham

\begin{abstract}
Injection molding is widely used to produce plastic components with large lot size. However, guaranteeing consistency and quality of parts in injection molding is challenging. Failures occur due to variation during injection cycles. Thus, real-time detection of failures will have a high impact on quality and productivity. This paper is focused on SHION, a cloud based Digital Twin supported by Al based control of process parameters. Process parameters and their interrelationship with quality failure were studied and used to generate models for real-time prediction of part quality. Two injection manufacturing lines in industry were chosen for data acquisition, implementation, and validation of the Digital Twin. While SHION successfully predicted faulty products in real time, adoption of traditional Cloud-centric loT approaches poses unforeseen practical challenges such as exposure to risk of losing data due to network issues and prohibitive cost of regularly transferring a large amount data to Cloud services.
\end{abstract}




\section{Introduction}

The plastic industry in Europe provided employment to more than 1.6 million and contributed $€ 28.8$ billion to the economy in 2018. Injection molding is the most used manufacturing process to produce plastic components. It includes four main stages: plasticization, injection, cooling, and ejection. Plastic material is fed by gravity from a hopper into a heated barrel. The plastic material melts as it moves forward in the heated barrel and then injected under high pressure into the molding. The melt is cooled in the molding to ensure dimensional stability and the part is ejected once it is cooled.

Part quality and dimensional integrity define injection moulding productivity. The quality of molded products depends on the complex interaction of multiple factors such as accuracy of an injection molding machine, quality or grades of plastic material, and process parameters . It is challenging to guarantee consistency and quality of produced parts in injection molding since quality failures may occur due to the variation of aforementioned factors during molding cycles. Therefore, process monitoring and anomaly detection of the produced parts are essential to ensure that the quality of the delivered products meet customers specifications. Shop-floor workers usually conduct quality check on each piece and quality department periodically check on some sample parts. Automatizing this process will spare material and time. Thus, ability to detect a production failure in real-time will have a high impact on production quality and productivity.

A Digital Twin (DT) plays an important role in enabling companies to gain insight on the actual processes at the shop floor. It is defined as a high-fidelity digital representation of the operational dynamics of its physical counterpart and requires near real-time synchronisation between the physical and digital counterparts [1]. The data flow between the physical and digital counterparts is the key characteristic of a DT. The adoption of DT by companies is expected to bring higher efficiency and accuracy as well as economics benefit. Current implementation of DT is largely still limited to conceptual work. Lu's et al. [1] only found a small number of DT application scenarios and none of them reported the imple- mentation of DT related to process monitoring in injection molding. This paper aims to fill this gap by reporting an actual implementation of a DT, so called SHION - Smart tHermoplastic injectION [2], in an industrial setting which allows capturing of real implementation issues, challenges, and benefits of a DT.

The remainder of the paper starts with section 2 which reports a literature review of DT. This is then followed by a context overview of SHION implementation and detailed description of $\mathrm{SH}$ ION architecture in section 3. Section 4 explains the implementation results. Section 5 provides discussion and reflection of SHION implementation whereas section 6 concludes the research work.

\section{Literature Review}

\subsection{Definition of DTs}

[3] reviewed existing DT definitions based on an industrial use case and defined a DT as a virtual dynamic representation of a physical system, which is connected to it over the entire life cycle for bidirectional (and automated) data exchange. They identified two aspects. First, physical twin transfers data and information from the real space to a virtual digital space when needed. Second, a DT identifies product or process oriented improvements, control demands based on the current situation, or predictions of the near future and sends them back to the real space so the physical product adapts accordingly. DTs are still in their infancy and many researchers are currently starting to derive appropriate concepts as a first step towards applying DTs in practice [4]. A DT can be seen as a collection of use cases contributing to an overall product life cycle management (PLM) strategy[3]. They are intended to be used through all the phases of the PLM [5]: requirements capture, product design, project / production planning, reliability of an engineering project, training or real-time decision-making [5] and customer support [6].

[5] identified tree types of DT based on product life cycle phases: Production (monitoring manufacturing/quality, forecast cycle times, etc.); Engineering (replace physical test, optimize product features through simulation, etc.); and Operation twins (monitoring use phase, product 
improvement, etc.).

A DT requires a bidirectional automated integration of data between physical and virtual worlds[7]. This is the feature that differentiates the DT concept from digital model (in which data integration is performed manually) and digital shadow (in which, data integration from physical world is automated but data from virtual world to physical world must be manually integrated).

\subsection{Architectures for implementing DT}

According to the definition of DT, any system architecture must be able to represent the physical product, the virtual product and the communication between them. However, the most important element is data. The elements of a DT and their interactions are reflected in Figure 1 adapted from [5].

Figure 1 also describes the requirements of the architecture of a DT: it must allow the high frequency of real-virtual data synchronization for fidelity guarantee; it must be possible to present a holistic view of available real data, synthetic data and knowledge of experts to a user; it must be able to automatically evaluate its behaviour, and enable real data feed to virtual models and continuous improvement of models by comparing their results with the physical space. Although DT community agrees on these features, the creation of a reference models for DT is still a challenge [8].

Figure 2 summarizes different proposals of DT Reference Architectures (RA) [9] [10] [11]. According to [9], the modules of a RA must offer their functionalities as services both to other components and external systems. It allows a distributed deployment of the architecture and, so it is possible a DT takes advantage of edge computing capacities [10]. Finally, this RA allows to implement the Reference Architectural Model Industrie (RAMI) 4.0 model[11].

Horizontal components of the proposed RA are related with: the bidirectional exchange of data between physical and virtual worlds (IoT Stack); storage of raw physical data, data generated by AI and simulation models and the models themselves (Data); interaction with existing systems such as ERPS, MESs, PLMs or CAD systems (Systems of Record); management of simulation models (Simulation Modelling); Big Data and ML and Deep Learning algorithms which can be used for real time monitoring, detecting abnormal patterns or recommending solutions to problems (Analytics and $\mathrm{AI}$ ); interacting with users of the Digital Twin (Visualization); and orchestration and management of the services implementing the given DT (Process Management).

Vertical components are perpendicular to horizontal ones providing access to services that must be used by the other to simplify the interaction between a DT components and of this with external systems (Integration); to make this integration secure (Security) and guaranteeing that the rules and policies of the company and laws of the place where the DT is located are complied with (Governance).

\subsection{Data driven DT}

Data driven modeling as the base of DT is becoming very popular[12]. These approaches apply IoT, Big Data and artificial intelligence, such as ML, technologies and methods for capturing, exchanging and transforming data between the virtual and physical parts of the twin.

In particular, ML models are used for monitoring and detect undesired behaviours such as faults in production. Faults prediction takes advantage of the fact that (appropriate) data obtained from physical twin can be a manifestation of both known and unknown physics, so ML models can account for the full physics and use it for predicting the errors[12].

While ML can be applied to predict behaviours it is also used to understand the structure of time series, reduce the number of variables to be considered, classify images, and create synthetic data that increases available training data sets and addresses a lack of data[12].

An advantage of Data-driven models is that they will improve with new data. Although training of data-driven modeling might have issues associated with instabilities, they are quite stable for making predictions[12] once models are fully trained.

\section{SHION Concepts}

\subsection{Context of SHION}

SHION was implemented within the scope of CloudiFacturing research project [13]. The mis- 


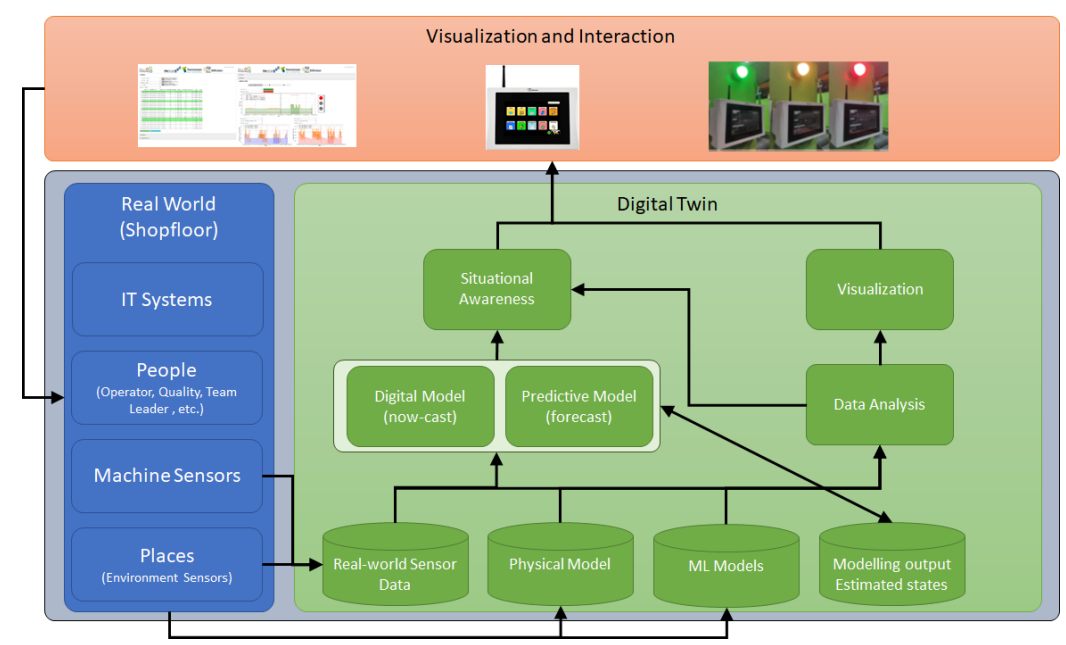

Figure 1. Digital Twin Data Workflows adapted from [5].

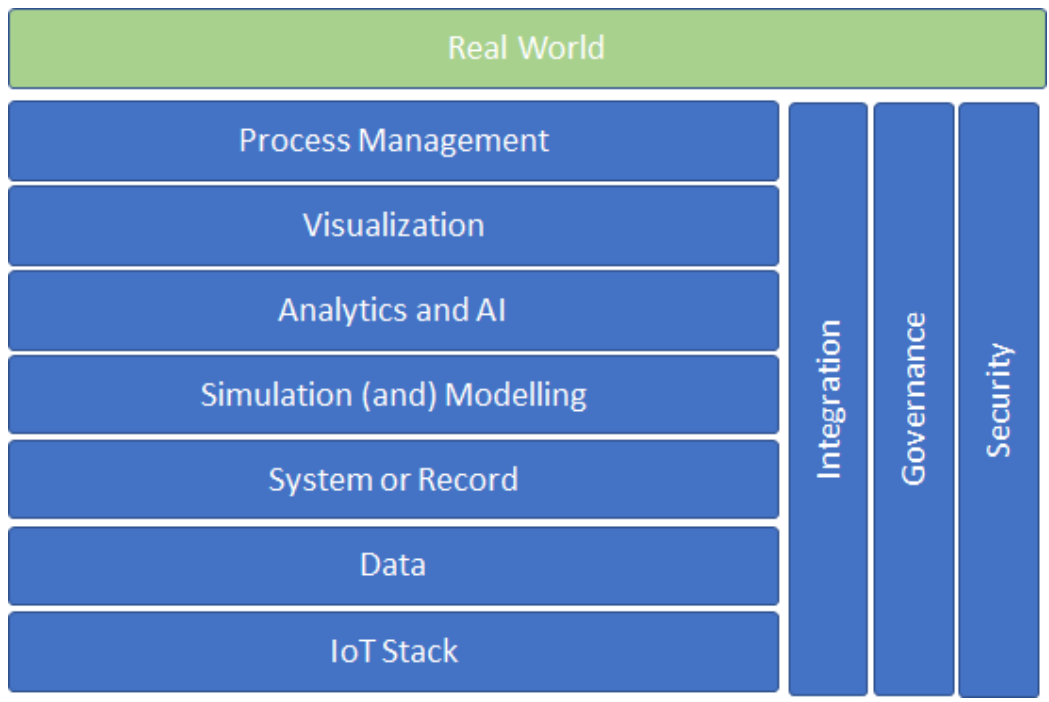

Figure 2. Digital Twin Reference Architecture.

sion of CloudiFacturing is to use Cloud/HPCbased modelling and simulation to foster the implementation of I4.0 in manufacturing Small and Medium Enterprises (SMEs). CloudiFacturing invited participation of third-party consortia to seek innovative use cases that fitted with the project's mission; and SHION was one of them. SHION consortium composed of: 1) a research centre, the Technological Institute of Aragón (ITAINNOVA), with expertise in big data and artificial intelligence services, 2) an independent software provider, BMS Vision (BMS), with expertise in offering a wide variety of industrial sensors, and 3) an end user, Thermolympic which specializes in manufacturing thermal injection plastics prod- ucts and provides the industrial problem to be solved.

\subsubsection{Industrial Problems at Thermolympic}

Maximizing production quality is crucial for Thermolympic. A machine operator visually reviewed all the produced parts, looked for general deviations and reported the defects. Additionally, a quality staff conducted statistical process control on some sample parts every four hours. While current quality rate was at $98.8 \%$, a further quality increase was important. Automated failure detection would spare material and time by avoiding reworking and reducing customer penalties because of delays and delivery of non- 
detected rejected parts to customer. The proposed approach was to take an advantage of cognitive technologies, in particular Machine Learning (ML)), to extract knowledge and generate a predictive model to detect when a defect in the production was going to happen. Constant evolution of the cognitive models generated would also be important. To realise SHION, information from the injection molding process itself as well as the context information such as environmental conditions, operators' reviews, quality laboratory inspections and piece weight were required. All data was collected via automatic and real-time devices using both IoT devices provided by BMS which was installed at Thermolympic. Instead of visually reviewing every part, an operator would only review the parts when SHION anticipated the presence of a possible fault. Consequently, the operator could perform additional tasks in between the possible fault notifications that they received. Thus, SHION would change the work nature of a machine operator, a team leader and and/or quality staff from corrective/contention to preventive/predictive flow.

\subsubsection{CloudiFacturing Platform}

CloudiFacturing integrates several existing software and hardware platforms for Cloud-based engineering and manufacturing. One of them is CloudBroker application. CloudBroker application uses the CloudBroker Platform which is a backend tool for the deployment, management and running of compute-intensive software on various Cloud infrastructures. For SHION implementation, persistent instance storage is required. If the connection to the instance was lost unexpectedly or a new instance had to be launched, the part of previous data stored on the initial instance had to be present on the new machine. Thus, a functionality to attach an Amazon S3storage to an instance as a disk was implemented in CloudBroker application to persist the changes kept in the mounted area.

\subsection{Architecture of SHION}

Figure 3 shows the final systems architecture of SHION which consists of two subsystems. The left box shows that the IoT and MES infrastructure are deployed on Thermolympic facilities and supported by BMS. This subsystem monitors ma- chine parameters and environmental conditions. It sends the parameters to the Cloud with a minimum injection cycle of $14 \mathrm{sc}$. It also interacts with machines operators both through the DU11 terminal of BMS and through a traffic light alarm system which allows operators to easily view the state of the machine and its products.

The right box shows the SHION intelligent modules which is deployed on a CloudBroker instance. SHION is powered by Argon, a Docker supported system supporting the customization of ITAINNOVA deployments. The Kong container controls the access to the REST interface of the predictive monitoring system, and to the Mosquitto messages queues. The Influxdb container stores time series of data processing from machine and environmental sensors and operators interactions with machines (e.g. declaring parts with defect and the cause of it). The MongoDB container stores Moriarty workflows that train predictive models (grey arrows) and supports predictions in real time (green arrows). These workflows record data received in the Influxdb repository. The Voilà container implements the interface supporting real time monitoring of the machine, displaying the prediction results as well as, allowing the creation of new models and the retraining of the new ones. This interface is used by team leaders and quality staff.

Real time monitoring system is fed through a REST API. Received parameters launch next workflow:

- The data is stored in an Influxdb database and prepared for predicting defects in the injected part. The parameters are identified by the machine identifier, the part identifier and the timestamp when parameters are taken.

- The predictions are performed using a model selected dynamically using the machine and the part identifiers. Firts machine and part model is search, if not found a machine model is search and, in the case no machine model is found, a general model is applied. The Acuracy of the models is related with the matching of machine and part with existin model being the general model the less accurate. SHION allows experts users (teams leaders and quality staff) to modify this default behaviour by manually assigning an already existing model 
to a machine-part pair or to a machine: existing know-how can be incorporated on production monitoring and failure prediction (i.e. when a part injection is moved from a machine to another machine).

- The results are communicated to workers together with the expected probability of the prediction using MQTT using the Mosquitto container. This messages are show using BMS Vision DU11 devices: it turns on required light (green, yellow, red) and a message is shown to operator requesting operator him/her feedback about the existence or not of a real failure and the cause of it. Figure 5)

While Figure 3 shows a monolitic architecture, the architecture is highly flexible. The versatility of Docker containers and the implementation of replicating data services allow the deployment in a scenario in which, modules in green dotted line is deployed on the Cloud and used on demand while the monitoring modules can be deployed on Thermolympic facilities. This can reduce the cost of the computational resources because high performance computing can be used on demand for training the models.

\subsection{ML prediction models creation and retraining}

The first step was to normalize and to create differentiated variables from the raw data setsc. For each variable and its successive measurements, the variation between the couple of values were calculated. A second transformation was applied to these derived measurements: moving averages, maximum dispersion in a time window and minimum value were calculated to obtain a summarizing set of statistical descriptive variables which was then normalized. The third step was to reduce the number of variables by employing Principal Component Analysis algorithm. In step

four, a final variable selection process was carried out using Random Forest algorithms with crossvalidation.

Once main injection features were selected, we trained different algorithms such as Artificial Neural Networks, Support Vector Machine and Tree-based models (Random Forest and Extra Random Forest). With the algorithms providing best results, optimal hyper-parameters sets were searched and used to train the prediction models for manufacturing lines.

\section{Implementation of SHION}

The findings showed that prediction models accuracy varies depending on the quality of the data given to the system. While SHION obtained lower precision rates in general models $(<75 \%)$, it achieved higher scores in models that were related to products $(>75 \%)$. These limitations were caused by the fact that the data collected from operators were not always precise or well timed.

SHION offers to the quality staff or to team leaders the possibilities of creating new models and of retraining existing models with new available data. The left side of Figure 4 show the screen where these actions can be performed. The user can set the following training parameters in oder to determine the data set use for training: Start date/end date; Machine/part; kind of algorithm to be used. These parameters are combined and they are sent to the cloud for obtaining new prediction models or newer versions of existing ones.

Once the new models or new versions of already existing models are available, the qualified users can check and compare them based on their quality (accuracy, retention, etc.). They can modify default model selection and they can also check in real time the current status of the machines (to see the parameters used in previous injections) together with the results of the failure predictions (see right side of Figure 4).

Some network issues were experienced during implementation. Synchronisation problems between the machines and the Cloud resources appeared due to the use and availability of CloudBroker resources and persistence instances. An alternative solution to provide optimal use of resources and functionality was adopted by setting up a two server model at Thermolympic. The prediction monitor service was deployed locally (running in persistence at 7 days 24 hours) and reserving the use of Cloud only for training purposes at selected periods. This alternative solution significantly reduced the use of bandwidth and risk of losing manufacturing data.

An iterative usability evaluation was also performed to ensure that SHION could be used 
Module 1 flow: Real Time Prediction

Module 2 flow: Model Training

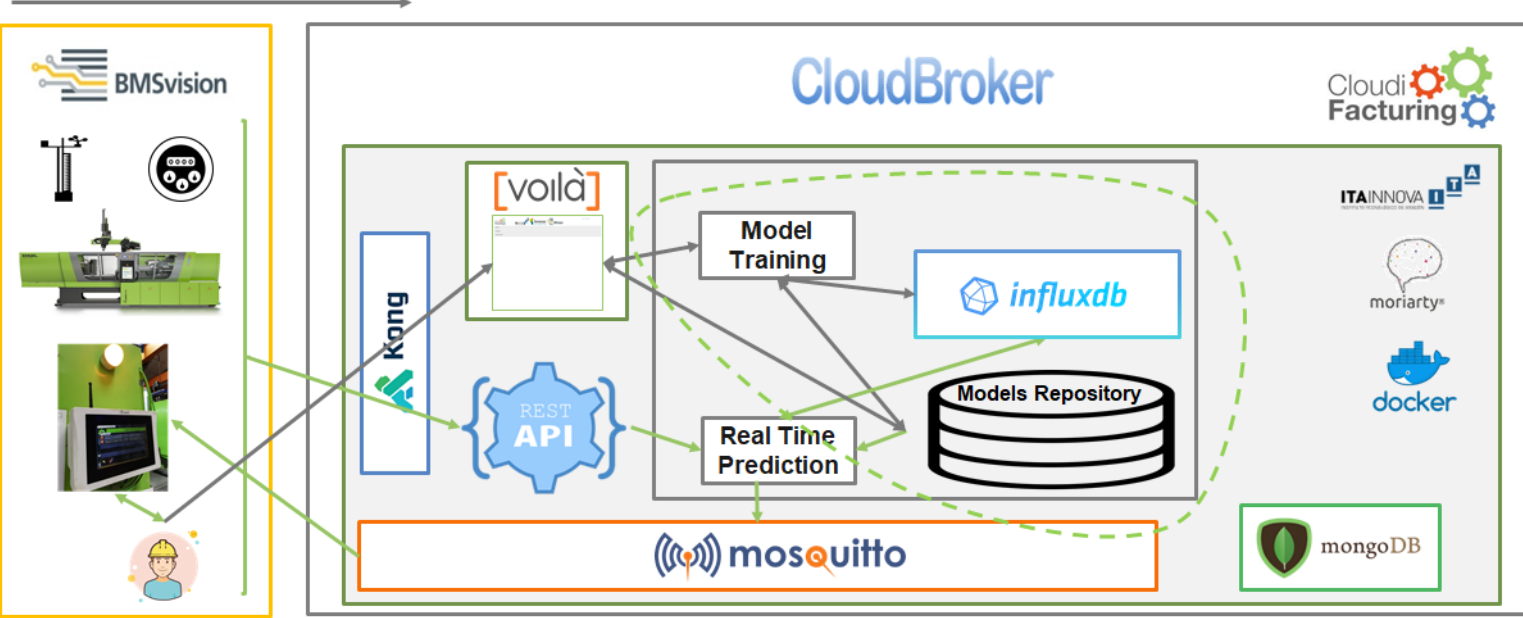

Figure 3. SHION architecture deployed in Cloud Broker.
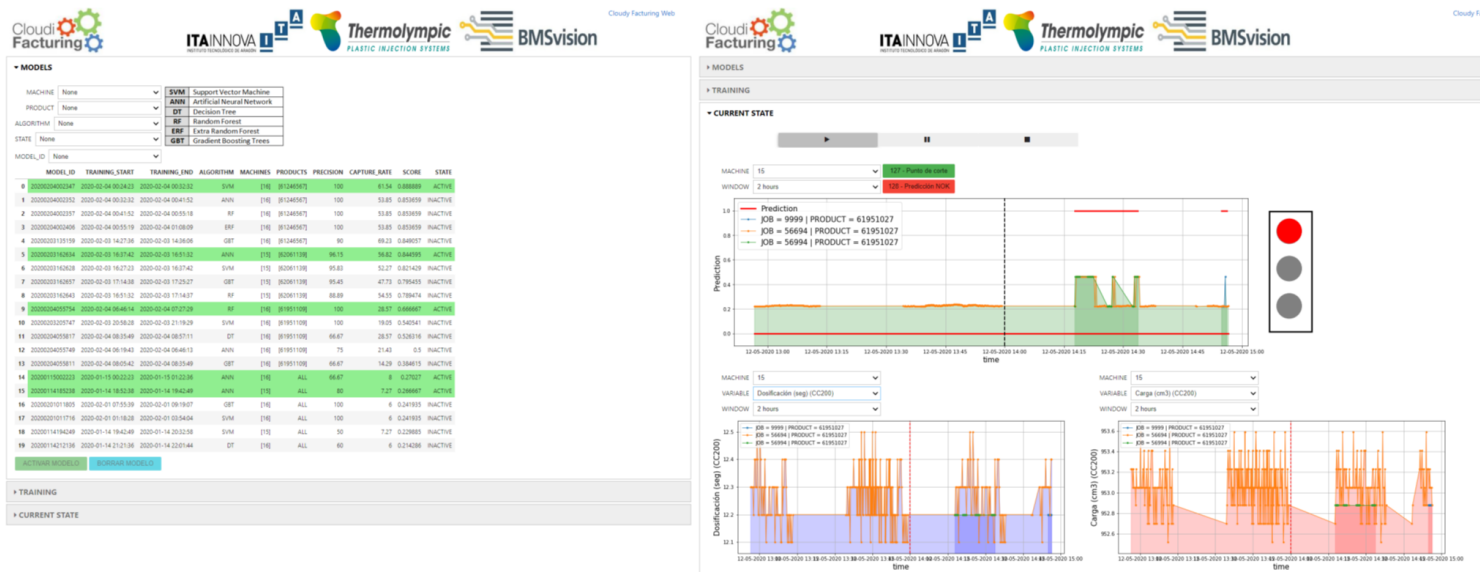

Figure 4. Left - Screen shots of the model management application (left) and of the real time monitoring application (right).
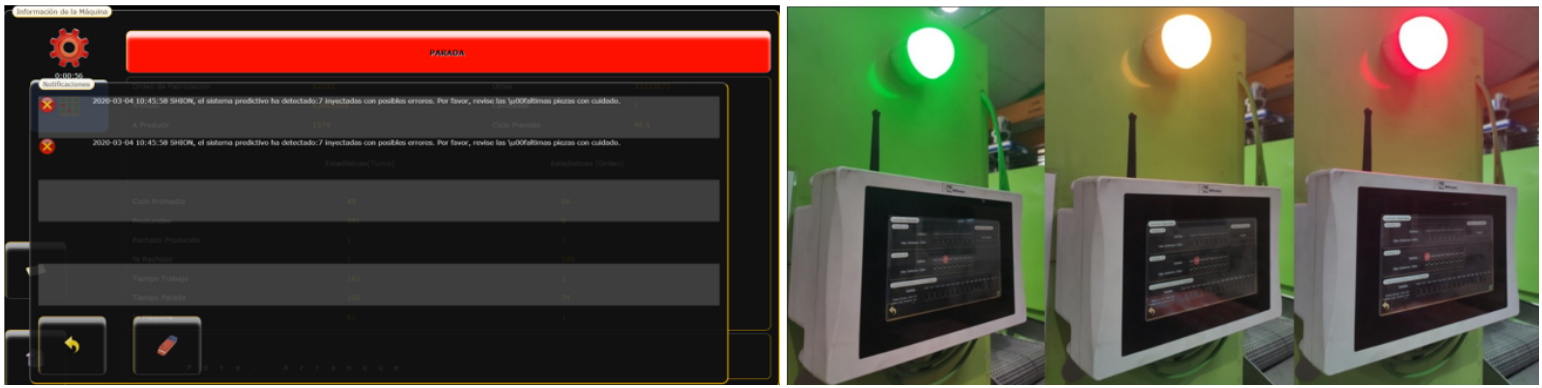

Figure 5. Left - Digital envelope received at human-machine interface at the shop floor; Right - Colour coded visual signals at the shop floor. 
effectively by users. A usability expert observed a quality manager and an injection molding operator while interacting and using SHION. Usability issues were identified and recommendations to resolve them were proposed and communicated to be acted on.

\section{Discussion}

SHION implementation demonstrated the capability to integrate real-time production intelligence data analytic on the shop-floor by providing feedback to both machine operator and to quality monitoring staff. Although many studies have demonstrated this capability in closed condition at labs, it is very rare to see its application in real industrial setting. During the K-Messe 2019, SHION was the only solution which showed a functioning solution that worked on actual production environments.

Nowadays, most of the new (injection) machines can provide smart (proprietary) modules (both on Cloud and on board). However, this may not be the case in most SMEs, which commonly utilise older type machines (between 15 and 30 years old), and thus limits their ability to adopt I4.0 solutions. Fortunately it is possible to send data to the Cloud for older machines by using standard interfaces (such as Euromap 83) which then allow additional solutions such as SHION to generate intelligent models to monitor the production line in real time and upgrade or improve the models by taking advantage of new data captured from the production line. SHION implementation required companies that already had an I4.0 basic setup like Thermolympic. For companies without I4.0 basic setup, SHION solution could be costly to be implemented as most of the cost of the solution was related to IoT readiness [14], which required purchasing hardware and software costing around $250000 €$, to provide data for the solution.

Collecting and analyzing information in real time while avoiding excessive data transfer and data processing delays is the core principle of I4.0. Cloud have traditionally been used to process IoT data since they provide cheaper and virtually unlimited computing power. However, the burden of uploading data to remote Cloud could lead to inefficient uses of bandwidth and energy, exposure to risk of losing data and/or services due to network issues and prohibitive cost of regularly transferring so much data over the Cloud. As we have experienced during SHION implementation, the traditional Cloud-centric IoT approach was required to shift toward a distributed model so we could take advantage of smart and programmable Cloud services at the network edge. These Cloud services on the network offered computing and storage capabilities on a smaller scale and provided benefits such as saving energy and network bandwidth consumption by avoiding continuously upload data to the Cloud. This also means reduction in communication delays and the overall size of the data that needs to be migrated across the Internet. As in the case of SHION, we employed a set of mechanisms to process data on behalf of the IoT device and effectively sending data to the Cloud only when more complex analysis is required. Thus, edge computing, fog computing and Cloud computing offer ideal technical solutions for different-level of DT requirements [15].

In SHION implementation, the created models were supervised models. While their accuracy is acceptable, it is highly dependent on machine operator availability to provide feedback on quality issues reporting data. The presence of unbalanced data can bias the results models, especially in the case where there is a lack of labelled data or data labelling is insufficient [12]. This could provide a barrier for improving the accuracy of models. However, the analysis we performed on data for creating the models showed us possible measures which could be implemented in the future to mitigate this risk, they are as follows:

- To include automatic detection of unexpected stoppage of machines: stop events are automatically logged and their causes should be declared by operators before restarting the machine.

- To detect undesired behaviour trends of machine parameters: use past events (part faults, machine stops, etc.) for detecting trends in parameters which conducted to them.

- To use previous (success) machine settings for a given part for recommending parameters when setting a new production bath or a new part (never injected before) is being setting up.

- To use operator feedback to model generated 
alarms for retraining the models and to support decisions of the quality manager about activating, deleting, retraining of a given model.

- To consider the use of unsupervised algorithms, such as self organized map, or clustering analysis algorithms, such as K-mean o $\mathrm{t}-\mathrm{SNE}[12]$.

- To improve the capabilities of the virtual twin by including interaction with physical plastic injection models for supporting Hybrid Analysis and Modeling.

To successfully implement a DT solutions, artificial model results must be presented in such a way that they are understandable [16] by users. Data presentation has to allow users perceiving the presented facts in the context they happen, easing taking decisions [17].

Understanding the ML models is also important to demonstrate they are not biased [12]. Explainable AI (XAI) is a very state-of-the-art trend and aims to explain models in an understandable way and trustworthy to users. XAI aims to answer questions about how and why certain results are obtained, allowing users to justify and explain their data-driven decisions [18] and, in some scenarios, assess legislation compliance [17].

\section{Conclusion}

SHION was aimed to provide real time detection of production failure in injection molding. It has successfully included human involvement in DT and built a functioning DT architecture which can be deployed both in server -based and edge based. SHION implementation has shown Cloud computing was more suitable for training/retraining and local installations for real-time solutions suit better because they were cheaper and less risky. Our implementation also showed that easily scale on resources and the processes were not required to run continuously (24hx7d). The implementation also showed the feasibility of utilising sensors available in market to implement DT.

\section{Acknowledgment}

This work was funded by the CloudiFacturing (Cloudification of Production Engineering for Predictive Digital Manufacturing) project, Project No. 768892.

\section{REFERENCES}

1. Y. Lu, C. Liu, K. I. Wang, H. Huang, and X. Xu,"Digital Twin-driven smart manufacturing: Connotation, reference model, applications and research issues," Robotics and Comput. Integrated Manufacturing, vol 61, 2019.

2. SHION experiment. CloudIFACTURING EXPERIMENT 10 - SHION: Smart tHermoplastic injectION, 2020. Accessed on: Oct. 30, 2020. [Online]. Available: https://www. $\{C\}$ loudifacturing.eu/exp-10- $\{\mathrm{S}\}\{\mathrm{H}\}\{\mathrm{I}\}$ $\{\mathrm{O}\}\{\mathrm{N}\}$-smart-thermoplastic-injection/

3. J. Trauer, S. Schweigert-Recksiek, C. Engel, K. Spreitzer and M. Zimmermann, "What is a Digital Twin?-Definitions and Insights from an Industrial Case Study in Technical Product Development," in Proc. of DESIGN 2020 Conf., Dubrovnik, Vol. 1, pp. 757-766.

4. W. Kritzinger, M. Karner, G. Traar, J. Henjes and W. Sihn, "Digital Twin in manufacturing: A categorical literature review and classification", IFAC-PapersOnLine, Volume 51, Issue 11, 2018, Pages 1016-1022, ISSN 24058963,doi:10.1016/j.ifacol.2018.08.474, 2018.

5. A. Stanford-Clark, E. Frank-Schultz, and M. Harris, What are Digital Twins?, 2019. Accessed on: Nov. 2, 2020.[Online]. Available: https://developer.ibm.com/technologies/ iot/articles/what-are-digital-twins/

6. F. Tao, J. Cheng, Q. Qi, M. Zhang, H. Zhang, and F. Sui. (2017). "Digital twin-driven product design, manufacturing and service with big data," Int. J. Adv. Manuf. Technol., vol. 94, nos. 9-12, pp. 3563-3576, Mar. 2017.[Online]. Available doi: 10.1007/s00170-017-0233-1.

7. W. Kritzinger, M. Karner, G. Traar, J. Henjes, and W. Sinn, "Digital twin in manufacturing: A categorical literature review and classification," IFACPapersOnLine, vol. 51, no. 11, pp. 1016-1022, 2018.

8. Y. Lu, C. Kevin, I. Wang, K. Huang, and H. Xu, "Digital Twin-driven smart manufacturing: Connotation, reference model, applications and research issues," Robotics and Computer-Integrated Manufacturing, vol. 61, pp. 101837, 2020.

9. A. J. H. Redelinghuys, K. Kruger, and A. Basson, "A sixlayer architecture for digital twins with aggregation," in SOHOMA 2019, Valencia(Spain), pp. 171-182.

10. Q. Qi, and F. Tao, "Digital twin and big data towards smart manufacturing and industry 4.0: 360 degree comparison,“ IEEE Access, vol. 6, pp. 3585-3593, 2018.

11. K. Schweichhart, Reference architectural model industrie 4.0 (RAMI 4.0). An Introduction, 2016. Accessed on: Nov. 2, 2020. Available online: http://w3devlabs.net/wp/wp-content/uploads/2017/ 05/a2-schweichhart-reference_architectural_model_ 
industrie_4.0_rami_4.0.pdf

12. Rasheed, Adil, Omer San, and Trond Kvamsdal, "Digital twin: Values, challenges and enablers from a modeling perspective," IEEE Access, vol. 8, pp. 21980-22012, 2020.

13. CloudiFacturing, CloudiFacturing Project Home Page, 2020. Accessed on: Oct. 30, 2020. [Online]. Available: https://www.cloudifacturing.eu/

14. Y. Turovets, and V. Konstantin, "Patterns of digitalisation in machinery-building industries: evidence from Russia," Eng. Manage. in Prod. and Services, vol. 11, no. 4, pp 7-22, 2019

15. Q. Qi, D. Zhao, T.W. Liao, and F. Tao, "Modeling of cyber-physical systems and Digital Twin based on edge computing, fog computing and Cloud computing towards smart manufacturing," in Proc. of ASME 2018.

16. S.D. Taylor, and E.P. White, "Automated data-intensive forecasting of plant phenology throughout the United States," Ecological Applications. Accessed on: Nov 8, 2020. [Online]. Available: https://doi.org/10.1002/eap. 2025

17. A. Holzinger, "From machine learning to explainable AI," in DISA 2018, pages 55-66.

18. R. R. Hoffman, S. T. Mueller, G. Klein, and J. Litman, "Metrics for explainable Al: Challenges and prospects," arXiv:1812.04608, 2018. [Online]. Available: http://arxiv.org/abs/1812.04608

Francisco J. Lacueva-Pérez has a MSc. Degree in Computer Engineering from the University of Zaragoza (Unizar). He works at ITAINNOVA since 2004 and he is currently working in the Big Data and Cognitive Systems team. He has experience in the planning, development and management of $R \& D$ projects both in the field of public (FP7, H2020, EUREKA, AVANZA, RETOS, CDTI, FET, etc.) and in private financing ones such asFACTS4WORKERS (H2020 6366778) and Experiment 10 of Cloudifacturing (H2020 768892 or agribusiness (GrapevineINEA/CEF/ICT/A2018/1837816). Nowadays, he does a doctorate to apply Big Data and Artificial Intelligence to improve the efficiency and sustainability of wine farms. Contact Francisco at fjlacueva@itainnova.es

Setia Hermawati is as a Research Fellow at Human Factors Research Group at the University of Nottingham. She obtained her PhD at Design School at Loughborough University. She has been involved in the conception, implementation and evaluation of new technologies for manufacturing systems. Her research Interest is in the ap- plication of user-centred design in novel technologies of manufacturing systems. Contact her at setia.hermawati@nottingham.ac.uk.

Pedro Amoraga is Computer Engineer and has worked at different positions related with quality (Quality Engineer and Quality Manager) at automotive sector, working directly for Tier 1 companies related to Ford, Mercedes, PSA, VolksWagen Contact Pedro at pedro@thermolympic.com.

icardo Salillas is a Physicist from the Unizar since 2016 and a master's degree in Mathematics, Statistics and Computing from the universities of the Basque Country and Zaragoza. He currently works as a data scientist in the Big Data and Cognitive Systems group at ITAINNOVA. Contar Ricardo at rsalillas@itainnova.es

Rafael del Hoyo, received the MSc. Degree in Physics and the Ph.D. degree in Artificial Intelligence at the Unizar. Currently, he is a project manager and responsible of Big Data and Cognitive Systems research line at ITAINNOVA. He participated in several projects related to Information Management and Artificial Intelligence for R\&D funded by the European Union in FP5, FP6, FP7, H2020 and Eureka-Celtic and National programs like Avanza. He was also a lecture of Software Engineering at Unizar and he is currently lecturer of the University of San Jorge about Intelligent Systems and information processing. Contact Rafa at rdelhoyo@itainnova.es.

Glyn Lawson is an Associate Professor within the Faculty of Engineering at the University of Nottingham, and member of the Human Factors Research Group. His research expertise includes human-centred development of new technologies. $\mathrm{He}$ is a Chartered Fellow of the Institute of Ergonomics and Human Factors and sits on the editorial board of Applied Ergonomics. Contact him at glyn.lawson@nottingham.ac.uk. 